[microresearch]

Diamond Open Access

\title{
Sum of all natural numbers
}

\author{
Open Mathematics Collaboration*†
}

February 18, 2020

\begin{abstract}
$1+2+3+4+5+6+7+8+9+10+11+12+\ldots=?$
\end{abstract}

keywords: series, natural numbers, infinite, divergence, convergence, infinity theorem

\section{Introduction}

1. $S=1+2+3+\ldots=$ ? $[1$

2. One trivial answer for (1) is $S=\infty$.

*All authors with their affiliations appear at the end of this paper.

†Corresponding author: mplobo@uft.edu.br | Join the Open Mathematics Collaboration 


\section{Sum of the natural numbers}

3. $S_{1}=1-1+1-1+1-1+\ldots=1 / 2[2]$

4. $S_{2}=1-2+3-4+\ldots$

5. $S=1+2+3+\ldots$

6. $2 S_{2}=S_{2}+S_{2}$

7. $2 S_{2}=(1-2+3-4+5-\ldots)+(0+1-2+3-4+\ldots)$

8. $2 S_{2}=1-1+1-1+1-\ldots=S_{1}$

9. $2 S_{2}=1 / 2$

10. $S_{2}=1 / 4$

11. $S-S_{2}=(1+2+3+4+5+6+7+8+9+\ldots)-(1-2+3-4+5-6+7-8+9-\ldots)$

12. $S-S_{2}=0+4+0+8+0+12+0+16+0+\ldots$

13. $S-S_{2}=4+8+12+16+\ldots$

14. $S-S_{2}=4(1+2+3+4+\ldots)$

15. $S-S_{2}=4 S$

16. $S-1 / 4=4 S$

17. $3 S=-1 / 4$

18. $S=-\frac{1}{12}$

\section{Final Remarks}

19. $S=1+2+3+\ldots=\{-1 / 12, \infty\}$

20. (19) supports the Infinity Theorem [3]. 


\section{Open Invitation}

Review, add content, and co-author this article [4,5]. Join the Open Mathematics Collaboration. Send your contribution to mplobo@uft.edu.br.

\section{Ethical conduct of research}

This original work was pre-registered under the OSF Preprints [6], please cite it accordingly $|7|$. This will ensure that researches are conducted with integrity and intellectual honesty at all times and by all means.

\section{References}

[1] Youtube, Numberphile. Astounding: $1+2+3+4+5+\ldots=-1 / 12$. https://youtu.be/w-I6XTVZXww

[2] Lobo, Matheus P. "The Convergence of Grandi's Series." OSF Preprints, 4 Jan. 2020. https://doi.org/10.31219/osf.io/aq5xz

[3] Lobo, Matheus P. "The Infinity Theorem." OSF Preprints, 4 Feb. 2020. https://doi.org/10.31219/osf.io/9zm6b

[4] Lobo, Matheus P. "Microarticles." OSF Preprints, 28 Oct. 2019. https://doi.org/10.31219/osf.io/ejrct

[5] Lobo, Matheus P. "Simple Guidelines for Authors: Open Journal of Mathematics and Physics." OSF Preprints, 15 Nov. 2019. https://doi.org/10.31219/osf.io/fk836

[6] COS. Open Science Framework. https://osf.io

[7] Lobo, Matheus P. "Sum of All Natural Numbers." OSF Preprints, 17 Feb. 2020. https://doi.org/10.31219/osf.io/yx28b 


\section{The Open Mathematics Collaboration}

Matheus Pereira Lobo (lead author, mplobo@uft.edu.br) $)^{1,2}$

${ }^{1}$ Federal University of Tocantins (Brazil); ${ }^{2}$ Universidade Aberta (UAb, Portugal) 